\title{
Improving the work function of the niobium surface of SRF cavities by plasma processing
}

\author{
P. V. Tyagi, M. Doleans, B. Hannah, R. Afanador, C. McMahan, S. Stewart, J. \\ Mammosser, M. Howell, J. Saunders, B. Degraff, S-H. Kim \\ SNS, ORNL, Oak Ridge, TN, USA
}

\begin{abstract}
:
An in-situ plasma processing technique using chemically reactive oxygen plasma to remove hydrocarbons from superconducting radio frequency cavity surfaces at room temperature has been developed at the spallation neutron source, at Oak Ridge national laboratory. To understand better the interaction between the plasma and niobium surface, surface studies on small samples were performed. In this article, we report the results from those surface studies. The results show that plasma processing removes hydrocarbons from top surface and improves the surface work function by 0.5 to $1.0 \mathrm{eV}$. Improving the work function of RF surface of cavities can help to improve their operational performance.
\end{abstract}




\section{INTRODUCTION}

Field emission is one of the critical issues in high gradient superconducting radio frequency (SRF) cavities that limits the cavity performance [1][2][3]. Previous studies on field emission in SRF cavities have concentrated on protrusions and particulate contaminations at the RF surface [4][5][6]. During surface studies at spallation neutron source (SNS), shallow coverage of hydrocarbons at the niobium surface has been systematically observed even after standard surface preparation such as chemical polishing followed by ultra pure water rinsing. Evidence of hydrocarbons on the RF surface has also been found in cavities [7].

Evidence shows that the hydrocarbon contaminations can enhance the field emission and may limit the cavity accelerating gradients. Plasma cleaning of SRF cavities is currently being pursued at the SNS, Oak Ridge National Laboratory (ORNL) [7]. Plasma cleaning of hydrocarbon contaminants is a well-established process in the semiconductor industries for cleaning of semiconductor wafers [8][9]. One of the attractive features of plasma processing for SRF cavities is that it can be apllied in-situ when cryomodules have been finally assembled and installed in accelerator tunnels. Other plasma processing for etching of niobium SRF cavities is currently being explored at J-lab [10][11].

Operating gradients of SNS six-cell high beta SRF cavities are presently limited by field emission and below the design value. An in-situ plasma processing technique at room temperature for these multicell cavities has been developed to remove hydrocarbon contamination [12][13][14]. Plasma in multicell SRF cavities is generated and tuned by using fundamental passband modes while a continuous gas flow is maintained. Neon is the primary gas for plasma ignition and tuning in a desired cell of the cavities. Once the plasma is ignited and tuned in a desired cell, oxygen is introduced into the cavity to oxidize hydrocarbon contaminants. The volatile by-products from the oxidation process are continuously pumped out. Plasma processed cavities have been tested at cold and showed significant reduction in the level of electron activities and improvement in their accelerating gradients [13][12][12].

In order to better understand the characteristics of the niobium surface after plasma processing, detailed surface studies on small samples were performed. This paper will discuss the results from those studies. Surface characterizations of niobium samples were done using secondary ion mass spectrometry (SIMS) for chemical analysis and with scanning kelvin probe technique for work function measurements. 


\section{IMPORTANCE OF WORK FUNCTION FOR SRF CAVITIES}

Field emission is one of the main factors that limits the accelerating gradients of SRF cavities. The field emitted current density is defined by the Fowler-Nordheim theory (eq.1) [15].

$$
j=a \frac{(\beta E)^{2}}{\varphi} \exp \left(\frac{-b \varphi^{\frac{3}{2}}}{\beta E}+\frac{c}{\varphi^{\frac{1}{2}}}\right)
$$

Where, $j$ is the current density, $\varphi$ is the surface work function, $E$ is the surface electric field, $\beta$ is known as the field enhancement factor and parameters $a=1.54 \times 10^{6}$, $\mathrm{b}=6.53 \times 10^{3}$ and $\mathrm{c}=10.4$ are constants.

Particulate contamination on rf surface can increase the field enhancement factor $\beta$ thus the field emitted current. To mitigate particle contamination, cryomodule assembly is for example done in clean room. It is believed that shallow coverage of hydrocarbons can lower the work function $\varphi$ of the niobium surface and also increase the field emitted current. In-situ plasma processing aims at removing hydrocarbon contamination and improving the work function of the cavity surface.

In the present work, a scanning kelvin probe system was systematically used to measure the work function of sample's surface [16][17]. This system operates on Lord Kelvin's principle and measures contact potential difference (CPD) between two metallic surfaces. During the measurements, the probe with known work function (gold plated and $2 \mathrm{~mm}$ diameter) vibrates and measures the CPD between sample's surface and probe's surface as a function of applied backing potential. The absolute work function of the sample's surface can then be calculated using following equation

$$
\varphi_{\text {sample }}=\varphi_{\text {probe }}+\mathrm{CPD}
$$

The accuracy level of the work function measurements were in the order of $\pm 0.1 \mathrm{eV}$.

\section{WORK FUNCTION SENSITIVITY TO TOP SURFACE COMPOSITION OF NIOBIUM}

In this article, top surface refers to the first monolayer of a material and near surface refers to several monolayers below the top surface. The work function for a surface is directly related to its composition. The reported work function of polycrystalline niobium is $4.3 \mathrm{eV}$ [18]. However, niobium is typically covered with its natural oxide of $\mathrm{Nb}_{2} \mathrm{O}_{5}$ with reported work function of $5.2 \mathrm{eV}$ [19]. Other factors such as crystal structure, oxide states and surface contaminations can significantly affect the work function of niobium surface [19][20][21]. Because work function is sensitive to surface conditions, the work function was measured systematically in the studies presented hereafter. In addition, 
chemical analysis of top surface and near surface was done by SIMS [22]. SIMS uses ion sputtering of the top surface and mass analysis of the secondary ions generated by the sputtering process. In the work presented here, the SIMS equipment [23] utilizes a primary beam of $\mathrm{Ga}^{+}$ions for sputtering of sample surface and a quadrupole mass filter for mass analysis. Chemical analysis by SIMS is very informative method especially for characterization of top surface composition but sensitivity of the signal measured for different species depends on various factors such as sputtering rate of different elements, ion escape probability etc. In the following, the signal of each species in each SIMS spectrum is normalized by the total intensity collected during the measurement.

\section{Work function and composition of a chemically polished niobium surface}

A niobium sample was buffered-chemically polished using standard acid mixture for cavity processing followed by rinsing in deionized water and drying under nitrogen flow in a clean room. The work function of chemically polished surface was measured at 4.5 $\mathrm{eV}$ which is significantly lower than the work function of $\mathrm{Nb}_{2} \mathrm{O}_{5}$ (niobium pentoxide). Chemical analysis by SIMS was performed to understand the lower work function. SIMS negative mass spectrum obtained for chemically polished niobium surface (fig. 1) shows mass peaks related to water, hydrocarbons, fluorine, and niobium oxides. The peaks at mass $12\left(\mathrm{C}^{-}\right)$and $13\left(\mathrm{CH}^{-}\right)$are fragments of hydrocarbon contaminants on the surface which can lower the work function.

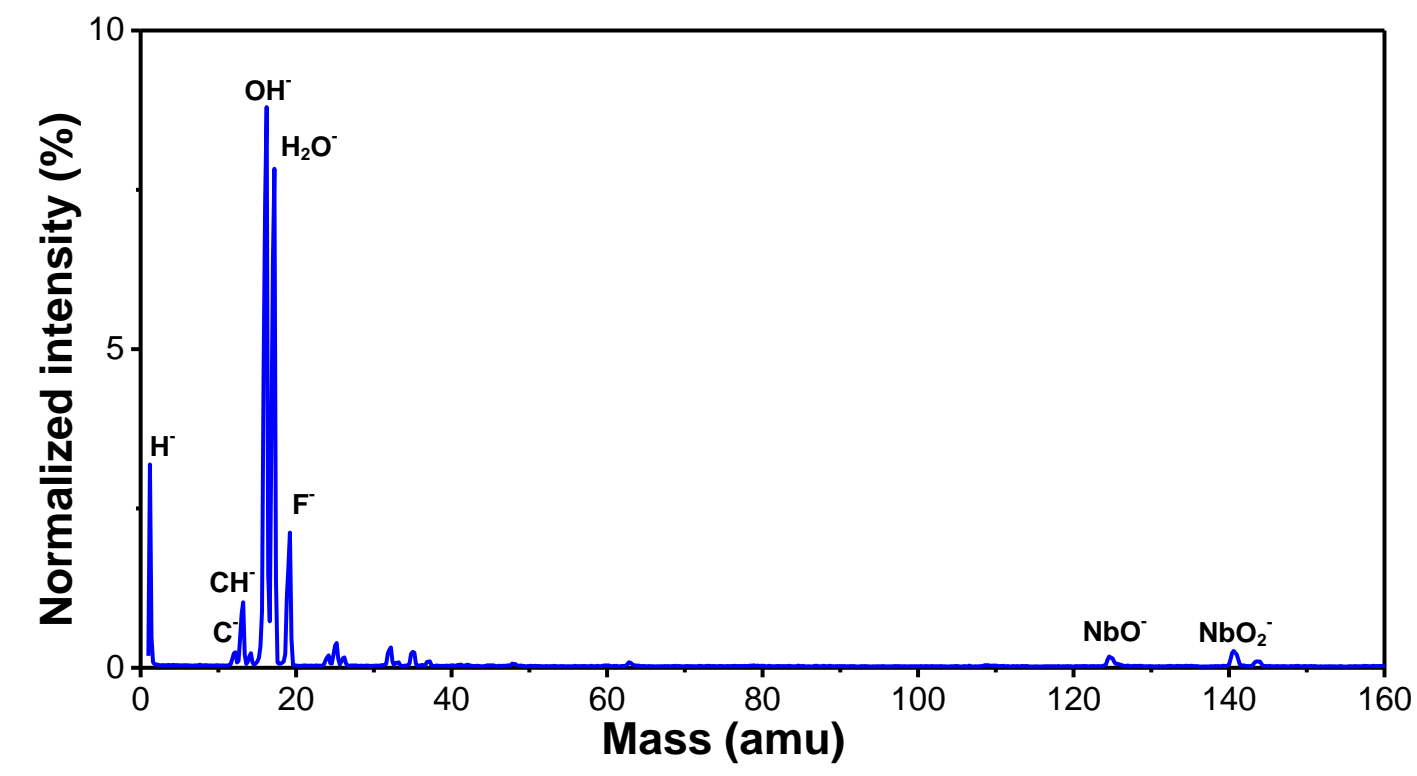

Figure 1: Negative SIMS spectrum of chemically polished niobium sample surface. 


\section{Work function and composition of a mechanically polished niobium surface}

Niobium samples were prepared by EDM wire cut out from a high purity niobium sheet and mechanically polished using a sample grinder/polisher [24]. Silicon-carbide abrasive paper was used for grinding and polishing. The samples were subsequently cleaned with isopropanol solvent and ultrasonically cleaned in deionized water for 15 minutes. The rms surface roughness of the mechanically polished samples was usually less than $1 \mu \mathrm{m}$. The work function of mechanically polished surface was measured typically at $4.8 \mathrm{eV}$ which is lower than the work function of $\mathrm{Nb}_{2} \mathrm{O}_{5}$. Similar to chemically polished samples, the SIMS negative spectrum of mechanically polished niobium samples shown in figure 2 also revealed the presence of hydrocarbons at the top surface.

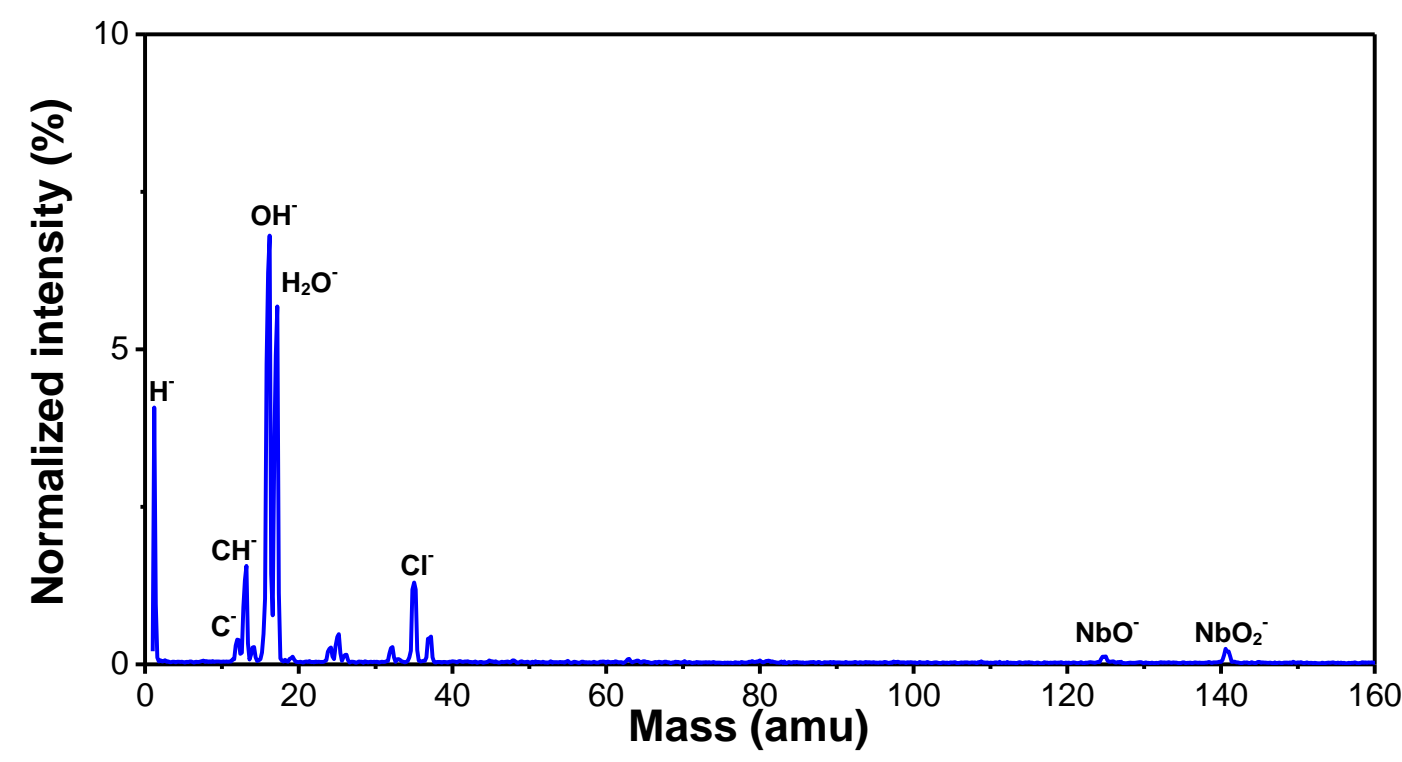

Figure 2: SIMS Negative spectrum of mechanically polished niobium sample surface. Similar to chemically polished niobium surface, hydrocarbon peaks were observed on the mechanical polished niobium surface. Small amount of $\mathrm{Cl}$ at top surface might have originated from sample preparations, grinding/polishing and handling.

\section{Lowering work function by artificial hydrocarbon contaminations}

In the SIMS spectra shown above, hydrocarbons were found at top surface and the work function in both cases was found lower than work function of $\mathrm{Nb}_{2} \mathrm{O}_{5}$. To investigate the possible effect of hydrocarbons on the niobium work function, mechanically polished samples were artificially contaminated using a methane plasma. The work function of those samples degraded from $4.8 \mathrm{eV}$ to $3.4 \mathrm{eV}$. This indicated that hydrocarbons can 
play a role in lowering the work function of the niobium surface. Development of a processing technique to remove hydrocarbons from the niobium surfaces and improve its work function was thus pursued.

\section{WORK FUNCTION IMPROVEMENT USING REACTIVE OXYGEN PLASMA TO REMOVE HYDROCARBONS}

\section{Studies on niobium samples}

The plasma processing studies (fig. 3) on niobium samples were performed using a 2.4 $\mathrm{GHz}$ microwave barrel plasma reactor [25]. The plasma reactor had two ports; one port was connected to a gas feed system and the other was connected to a vacuum pumping cart. The gas feed system used variable leak valves to adjust the gas mixture, pressure and flow to the plasma reactor and pirani gauges were used to monitor the process gas pressure in the plasma reactor. A residual gas analyzer (RGA) was used for analysis of the gases pumped out during plasma processing.

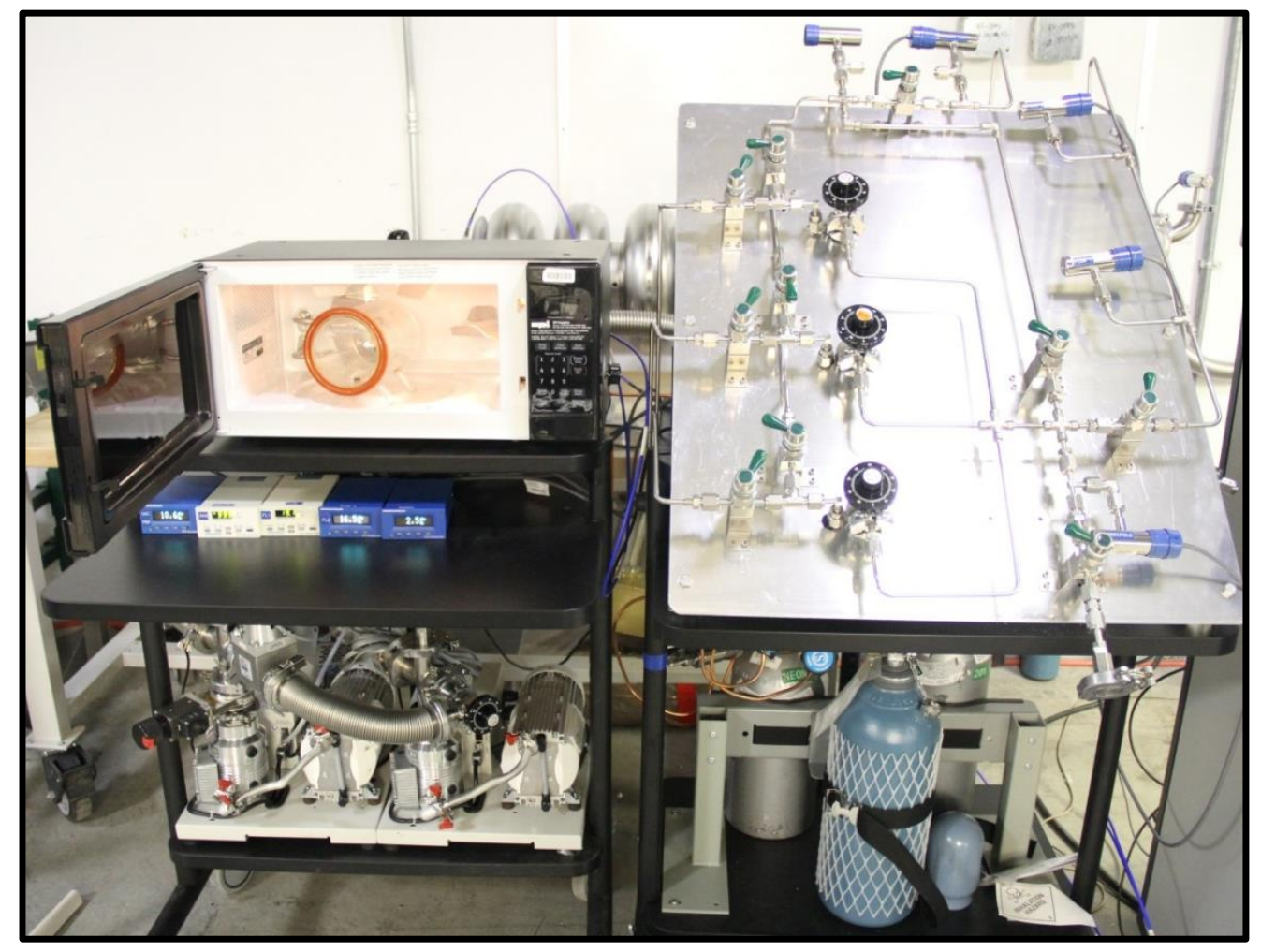

Figure 3: Microwave barrel plasma reactor with gas feed system.

Similar to plasma processing done in cavities, a mixture of neon and oxygen was used for the process gas. Typically, a base pressure of neon gas in the range of 100-200 
mtorr and 100 watts of microwave power were used for plasma ignition. For removing hydrocarbons, oxygen was introduced in the plasma; the oxygen concentration was in the order of a few percents (up to $2 \%$ ) of the neon pressure. To avoid significant heating of the plasma reactor and sample during plasma processing, short plasma cycles of 30 seconds were used. Figure 4 shows a neon plasma in the plasma reactor.

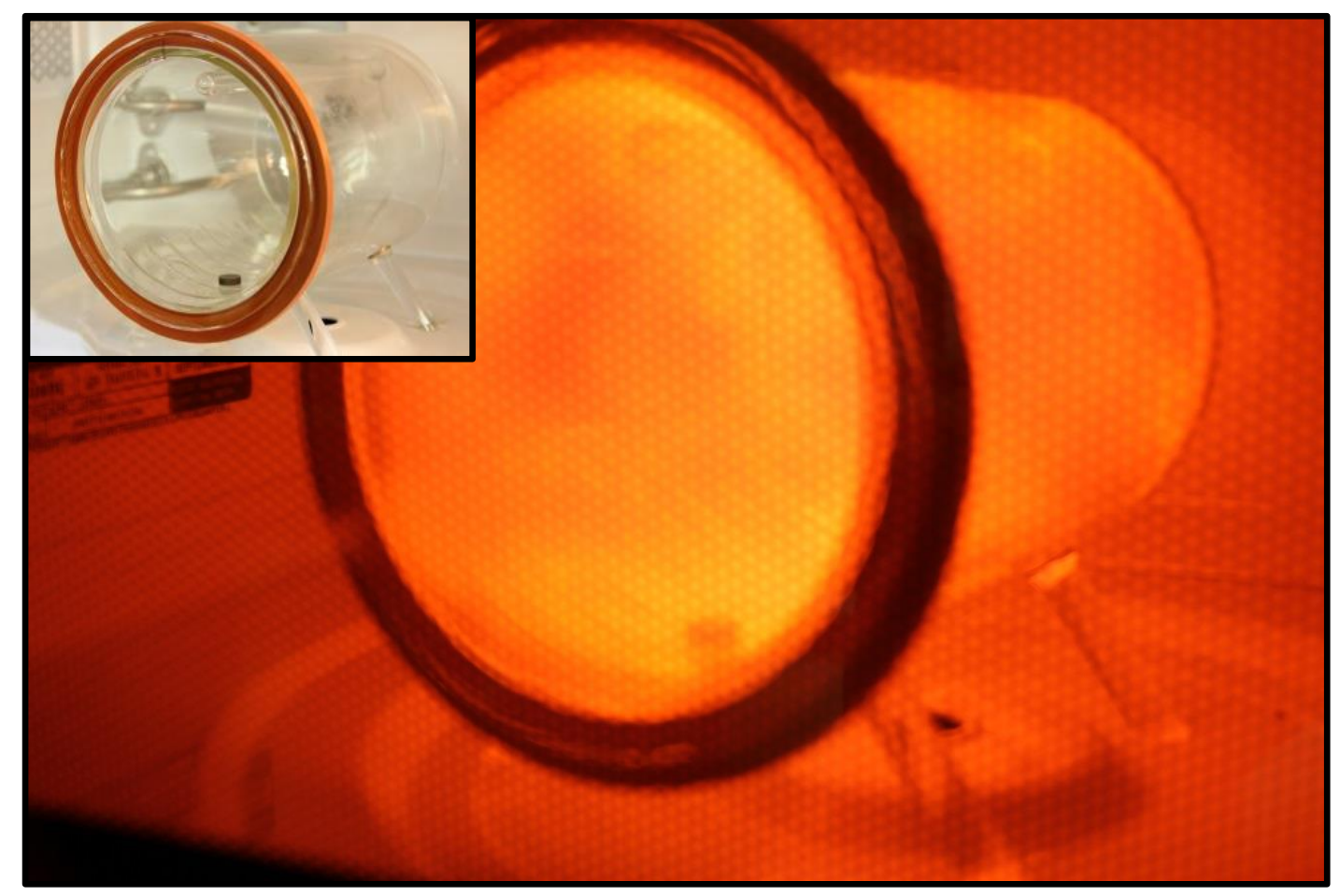

Figure 4: Neon plasma ignited in the plasma reactor (inset shows sample inside the barrel with no plasma).

To study the removal of hydrocarbons by the reactive oxygen plasma, SIMS measurements on mechanically polished sample were done before and after plasma processing in the plasma reactor. A total of 5 minutes of active plasma processing (10 plasma cycles) was used over an hour duration. The results from the SIMS measurements are shown in figure 5. Mass peaks of $\mathrm{C}^{-}$and $\mathrm{CH}^{-}$were reduced after the plasma processing confirming that reactive oxygen plasma was effective to remove hydrocarbons contamination from the niobium surface. Hydrogen peak was also reduced after plasma processing. 


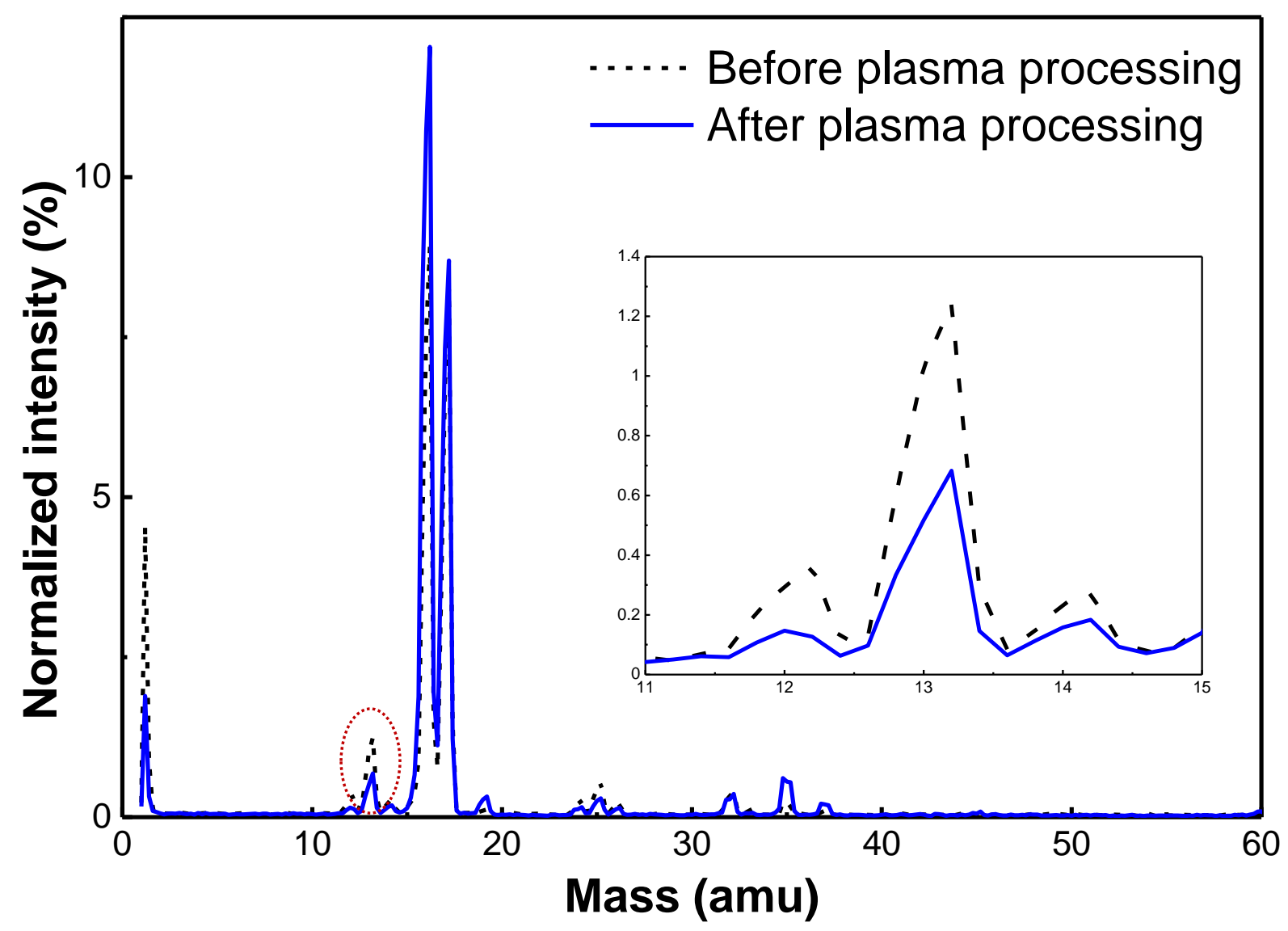

Figure 5: Negative mass spectrum of niobium surface before and after plasma processing. Inset shows the magnified view of $\mathrm{C}^{-}$and $\mathrm{CH}^{-}$peaks.

To measure the effect of reactive oxygen plasma on work function; scanning kelvin probe measurements were also performed on niobium samples. An increase in the niobium work function in the range of 0.5 to $1.0 \mathrm{eV}$ was observed after the plasma processing.

From the surface studies presented above it can be concluded that processing using reactive oxygen plasma removes hydrocarbons and improves work function of niobium surface.

Because oxygen has a strong affinity with niobium, the possible thickening of the oxide layer by the plasma processing was also investigated. In this regard, weight measurements on 25-mm diameter samples were performed before and after plasma processing. No change in the mass of the sample was observed within the measurement limitation of $\pm 10 \mu \mathrm{g}$ that corresponds to $\pm 8 \mathrm{~nm}$ for oxygen addition as $\mathrm{Nb}_{2} \mathrm{O}_{5}$. 


\section{Studies in SRF cavities}

Tests were conducted to confirm that reactive oxygen plasma can remove hydrocarbons from the surface of a cavity. Similar gas feed system described above was used for cavity processing. A residual gas analyzer (RGA) was used for monitoring gases pumped out of the cavity during the plasma processing. RGA data for some selected masses obtained during the plasma processing of a SNS high beta cavity are shown in figure 6 . The fluctuation in oxygen in the RGA data corresponds to oxygen introduction after plasma is ignited in a desired cell and stoped oxygen flow after processing is done (as described in the introduction part). The six cells of the cavity were processed sequentially and are marked with corresponding numbers in figure 6 . The position of plasma was confirmed with the help of cameras mounted at both the ends of the cavity. Mass peaks of $\mathrm{H}_{2} \mathrm{O}, \mathrm{CO}$ and $\mathrm{CO}_{2}$ were observed that correspond to the volatile byproducts of oxidation of the hydrocarbons by the plasma. For each cell, the partial pressure of the byproducts increased initially and then decreased over the time as hydrocarbons were progressively removed from the cavity surface during the plasma processing.

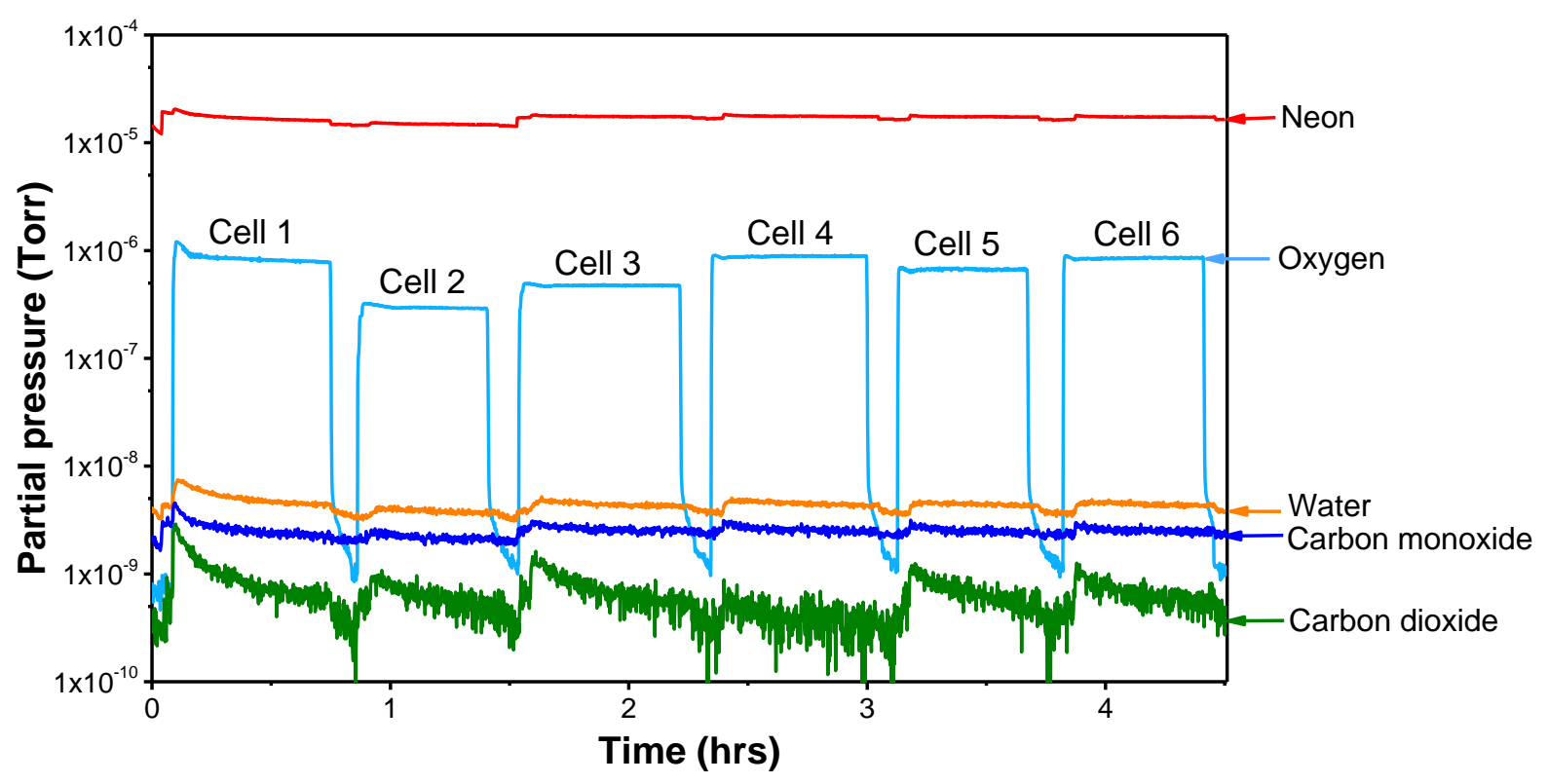

Figure 6: RGA data obtained during the plasma processing of a six-cell SNS high beta cavity. Cell numbers are shown in the figure.

The amount of hydrocarbons removed by the plasma processing can be estimated based on the flow of process gas and the partial pressures of the volatile byproducts. The number of moles per unit time for a continuous $P V$ gas flow is calculated using the ideal gas law [26] 


$$
\text { ? }=\frac{Q_{P V}}{R T}
$$

Where $Q_{P V}$ is the $P V$ gas flow rate in mbar'l/s, $R$ is the gas constant in mbarl/mol' $\mathrm{K}, T$ is the gas temperature in $K$ and $\dot{r}$ is the number of moles per second. The $Q_{P V}$ of the process gas can be estimated from the pressure drop over a length of gas line with known conductance. The number of moles per unit time for the volatile byproducts can be calculated from the ratio of their partial pressures to that of the process gas. The total amount of hydrocarbons can then be calculated by integration over the duration of the plasma processing.

To validate this methodology, a study on niobium sample with artificial hydrocarbon contamination was conducted using a mixture of neon and oxygen as the process gas. Experimental details can be found in the [27]. In this study the flow of process gas was dominated by neon and was $0.20 \mathrm{mbar} \cdot \mathrm{l} / \mathrm{s}$ which corresponds to $\dot{n}=8 \times 10^{-6} \mathrm{~mol} / \mathrm{s} \mathrm{using}$ equation 3. The total amount of carbonaceous byproducts was estimated from the partial pressures measurement in [27] and found equal to $2.9 \times 10^{-5} \mathrm{~mol}$. This coresponds to $350 \mu \mathrm{g}$ of carbon.

The amount of hydrocarbons removed by plasma processing was directly measured using an analytic mass balance and found equal to $210 \mu \mathrm{g}$ which is in reasonable agreement with the estimate using partial pressure measurements of the byproducts.

Hence, the latter method can be used to estimate the total amount of hydrocarbon contamination in a cavity during plasma processing. The amount of hydrocarbons removed from a cell of a cavity is typically equivalent to a fraction of a monolayer to a few monolayers.

Results from the studies of plasma processed cavities confirmed that the reactive oxygen plasma removes hydrocarbons from the cavity surface. Based on the results from studies on small samples, it is inferred that the work function of cavity surface is improved by plasma processing. In addition, volatile byproducts of reaction can be monitored during plasma processing and used to estimate how much contaminants were removed.

\section{EVOLUTION OF WORK FUNCTION IN VACUUM AT ROOM TEMPERATURE}

\section{Observation on niobium samples}

Sample studies showed that reactive oxygen plasma processing removes hydrocarbons and improves work function of the niobium surface. It was investigated if the increase in the work function could be sustained when the surface is kept in vacuum. For this, 
several niobium samples were plasma processed over a short period of time in the microwave barrel plasma reactor and their work function all increased by about $0.7 \mathrm{eV}$. Another sample was then plasma processed in the same manner but kept in the barrel vacuum for a few hours. The work function of this sample was then measured and found to be identical to its initial level before any plasma processing was applied. This showed that the work function can degrade even when a plasma processed surface is kept in vacuum at room temperature.

It was investigated if lateral migration [28] of contaminants from a non-plasma-cleaned region to a plasma-cleaned region could be responsible for such a degradation of the work function. A niobium sample of $25-\mathrm{mm}$ diameter was plasma processed with a mask of $10 \mathrm{~mm}$ diameter at its center. The work function of the plasma cleaned outer part of the niobium sample was measured at $5.5 \mathrm{eV}$ while it was $4.8 \mathrm{eV}$ at the masked region of the sample. The work function at the outer part of the sample was monitored over a few hours and no significant degration was measured. This study indicated that lateral migration does not play a significant role in the degradation of the work function when a plasma processed surface is kept in vacuum at room temperature.

SIMS measurement was then perfomed to understand how the change in top and near surface composition could explain the observed change in the work function. Since work function improvement by plasma processing was correlated with the removal of hydrocarbons from the surface, the recovery of hydrocarbons on the surface was investigated as a possible cause for work function degration when the surface was kept in vacuum.

Thus SIMS depth profile of $\mathrm{CH}^{-}$was obtained for a small sample before and right after 5 minutes of plasma processing and after keeping the sample an additional 15 minutes in the high vacuum of the instrument. The results are shown in figure 7 . As seen in figure 7 , the concentration of hydrocarbons was highest at top surface before plasma processing. Right after the processing, the concentration of hydrocarbons significantly decreased at top surface but not underneath. This shows that the plasma is effective to remove top surface contaminants only. After waiting 15 minutes in high vacuum the concentration of hydrocarbons at the top surface increased to its initial level while the concentration below the top surface decreased. This indicates that near surface hydrocarbons can migrate to top surface over time which could be one of the explanations for the observed degration of work function in vacuum. 


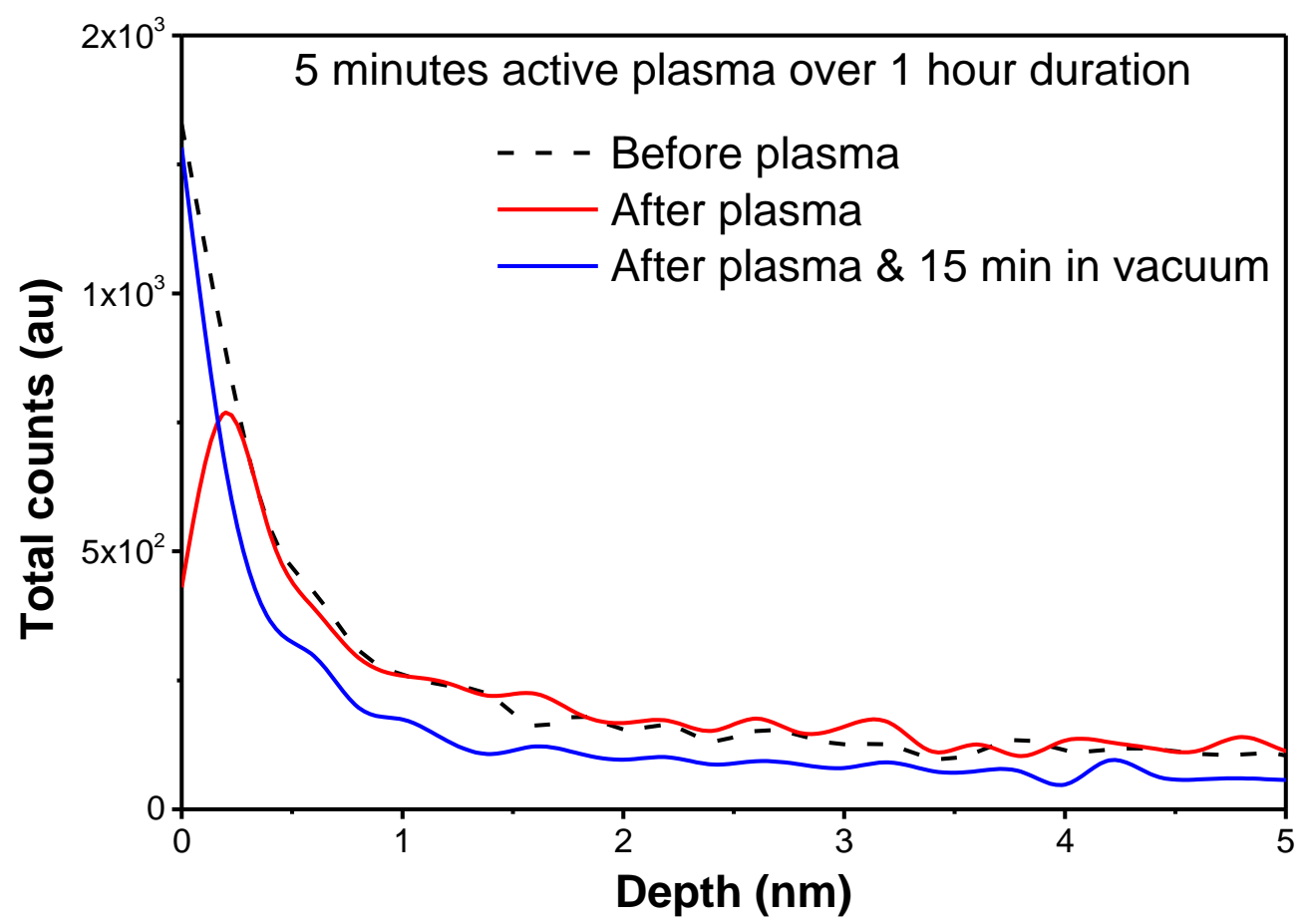

Figure 7: SIMS depth profile of $\mathrm{CH}^{-}$obtained for a small sample before and right after 5 minutes of plasma processing and after keeping the sample an additional 15 minutes in the instrument vacuum chamber.

\section{Observation in SRF cavities}

Removal and recovery of hydrocarbons at the top surface (rf surface) was also observed in high beta cavities when plasma processed and then kept under high vacuum at room temperature. As shown in figure 6 , removal of hydrocarbons in a cavity was indicated by the partial pressures of the byproducts of hydrocarbons oxidation. Those partial pressures were higher at the beginning of plasma processing and reduced at the end as the top surface became depleted of hydrocarbons. When that cavity was kept under high vacuum at room temperature and plasma processed again sometime later, the partial pressures of the byproducts had recovered. This indicates that some hydrocarbons had returned to the top surface. Figure 8 summarizes the partial pressure for one of the byproducts, carbon dioxide, for the sequence described above.

Observations on small samples and SRF cavities indicated that hydrocarbons were removed from the top surface by plasma processing and can recover even when the surface is kept under high vacuum at room temperature. Correspondingly, the work function increases after plasma processing and degrades after the hydrocarbons recover at the top surface. 


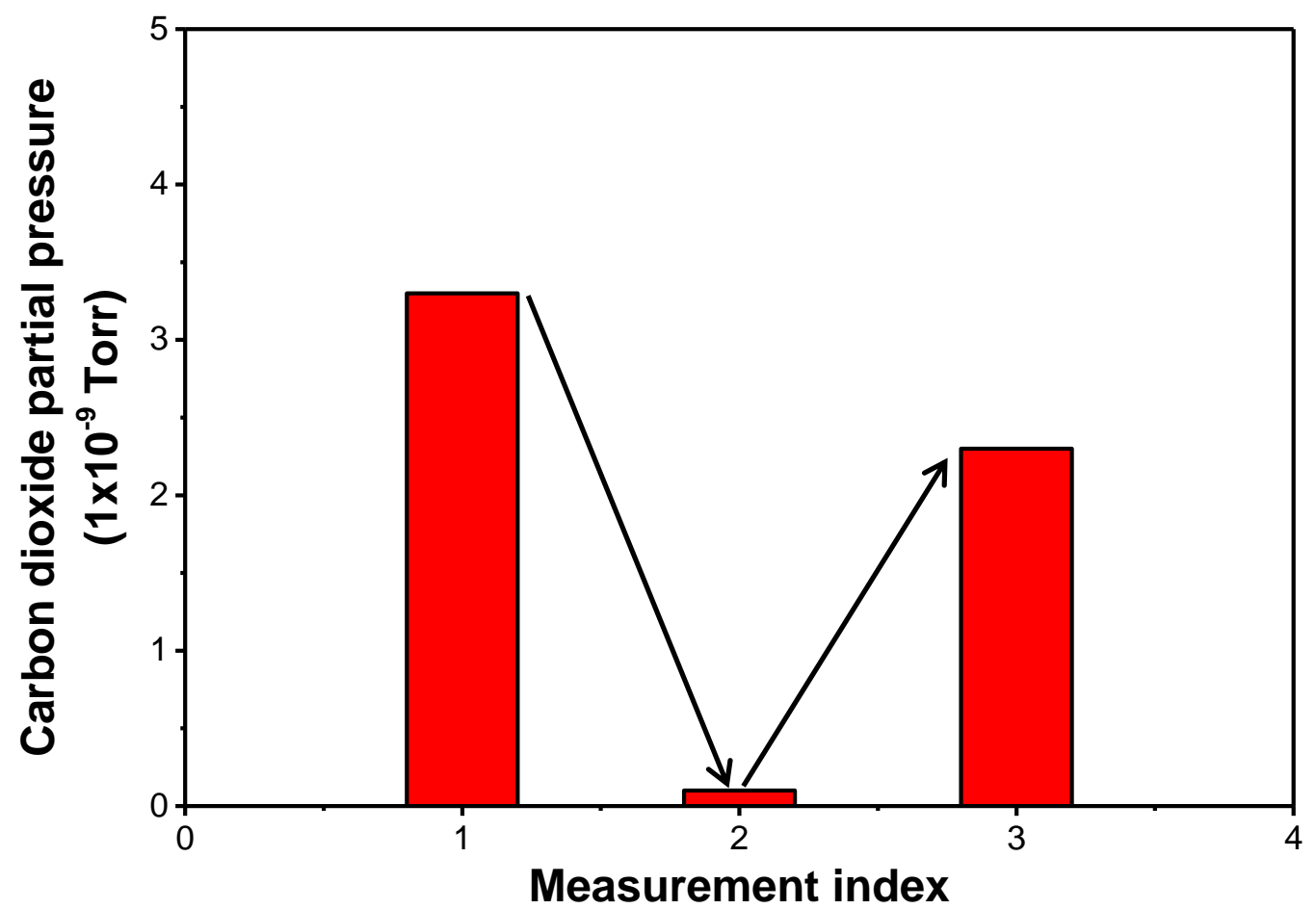

Figure 8: Partial pressure of carbon dioxide measured during an initial and subsequent plasma processing of a cavity. Measurement 1: At the beginning of the initial plasma processing, 2: At the end of the initial plasma processing and 3: At the beginning of the subsequent plasma processing. The cavity was kept under high vacuum at room temperature after the initial plasma processing.

\section{SUSTAINING HIGHER WORK FUNCTION}

As seen in figures 7 and 8 , the hydrocarbons can recover at the top surface after plasma cleaning and degrade the work function even when the surface was kept under vacuum at room temperature. To mitigate such a hydrocarbon recovery, longer and additional plasma processing cycles were explored.

As an example, a niobium sample was plasma processed with 15 minutes of active plasma over a 3 hours duration. Figure 9 shows the SIMS depth profile of $\mathrm{CH}^{-}$for this sample before and right after the plasma processing and after keeping the sample an additional 15 minutes in the instrument vacuum chamber. As seen in figure 9, the concentration of hydrocarbons was significantly decreased at top surface and near surface right after plasma processing. This is significantly different from the results obtained with the plasma processing over shorter duration shown in figure 7 where the concentration of hydrocarbons only decreased at the top surface. The reactive oxygen plasma processing intrinsically only interacts with top surface. But the longer duration enables hydrocarbons from near surface to migrate to the top surface where they can be removed by the plasma. Because of this effect, hydrocarbons recovery at the top surface for the plasma processing over longer duration was reduced after waiting 15 minutes in high vacuum. Hence, a few plasma processing cycles with waiting periods in 
between can be an effective methodology to remove hydrocarbons from top and near surface regions and mitigate hydrocarbons recovery at the top surface.

To evaluate how this methodology can mitigate the work function degradation in vacuum at room temperature, a specific work function study was performed. The experimental details of this study are summarized in table 1 and results are shown in figure 10.

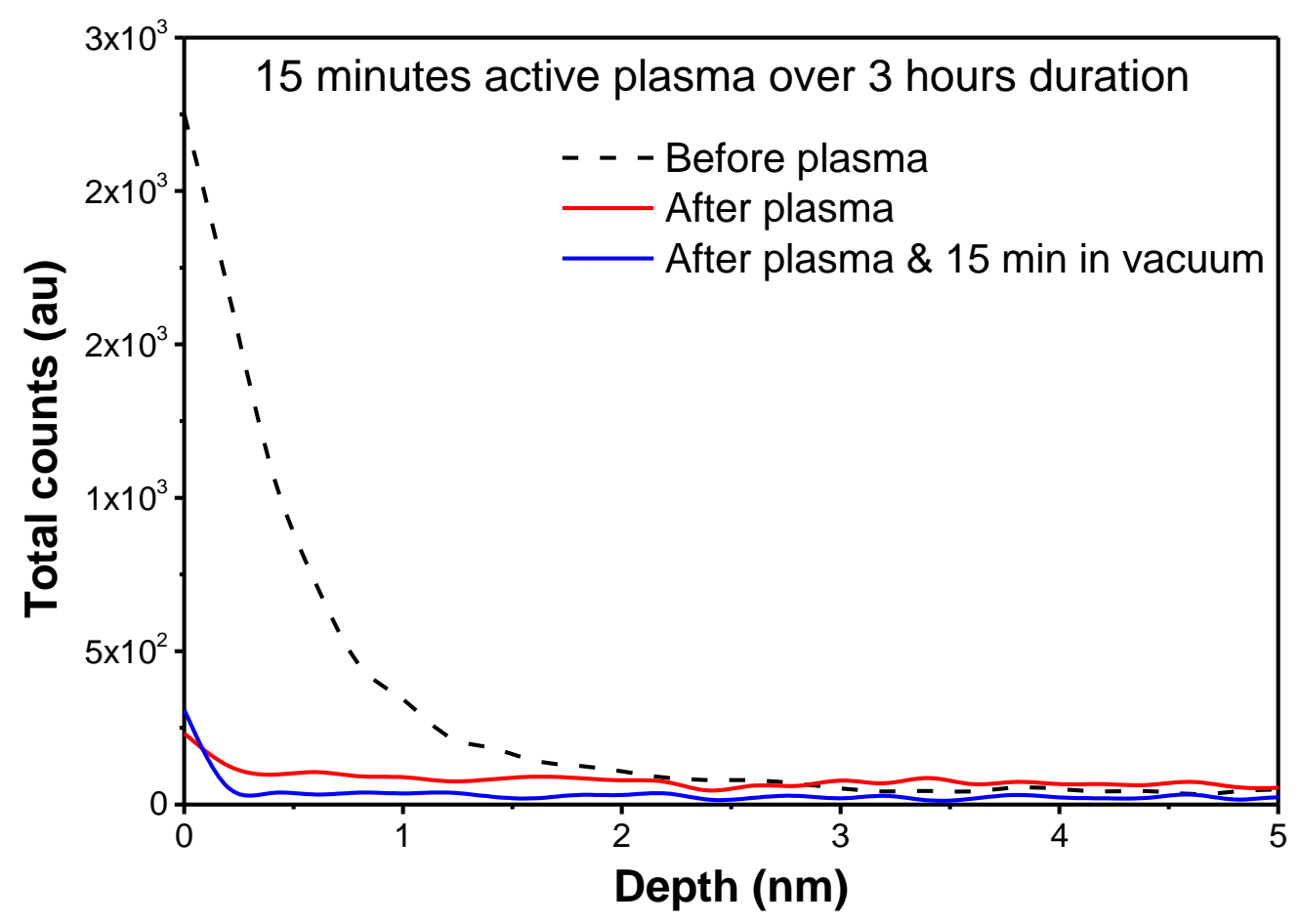

Figure 9: Comparison of $\mathrm{CH}^{-}$depth profile at niobium surface before plasma, after longer plasma processing and 15 min waiting in vacuum.

In this study, the workfuction of a sample was measured after three consecutive cycles of plasma processing and waiting periods in high vacuum at room temperature. As seen in table 1 and figure 10, the work function was always increased right after plasma processing and degraded after waiting periods in vacuum. However, accumulating plasma processing cycles with waiting period in between helps to sustain higher work function in vacuum at room temperature. 


\begin{tabular}{|c|c|c|c|}
\hline \multicolumn{2}{|c|}{ Sequence } & Experimental details & Work function (eV) \\
\hline 0 & & Initial condition & 4.8 \\
\hline \multirow[t]{2}{*}{1} & a & $\begin{array}{l}8 \text { minutes of active plasma over } 1.5 \text { hours total } \\
\text { plasma }\end{array}$ & 5.3 \\
\hline & $\mathrm{b}$ & 15 hours waiting in vacuum & 4.7 \\
\hline \multirow{2}{*}{2} & a & $\begin{array}{l}\text { Additional } 17 \text { minutes of active plasma over } 3.5 \\
\text { hours total plasma }\end{array}$ & 5.4 \\
\hline & $\mathrm{b}$ & 15 hours waiting in vacuum & 5.2 \\
\hline \multirow[t]{2}{*}{3} & a & $\begin{array}{l}\text { Additional } 8 \text { minutes of active plasma over } 1.5 \\
\text { hours total plasma }\end{array}$ & 5.4 \\
\hline & $\mathrm{b}$ & 24 hours waiting in vacuum & 5.2 \\
\hline
\end{tabular}

Table 1: Summary of the experimental conditions for work function studies experiments after subsequent cycles of plasma processing.

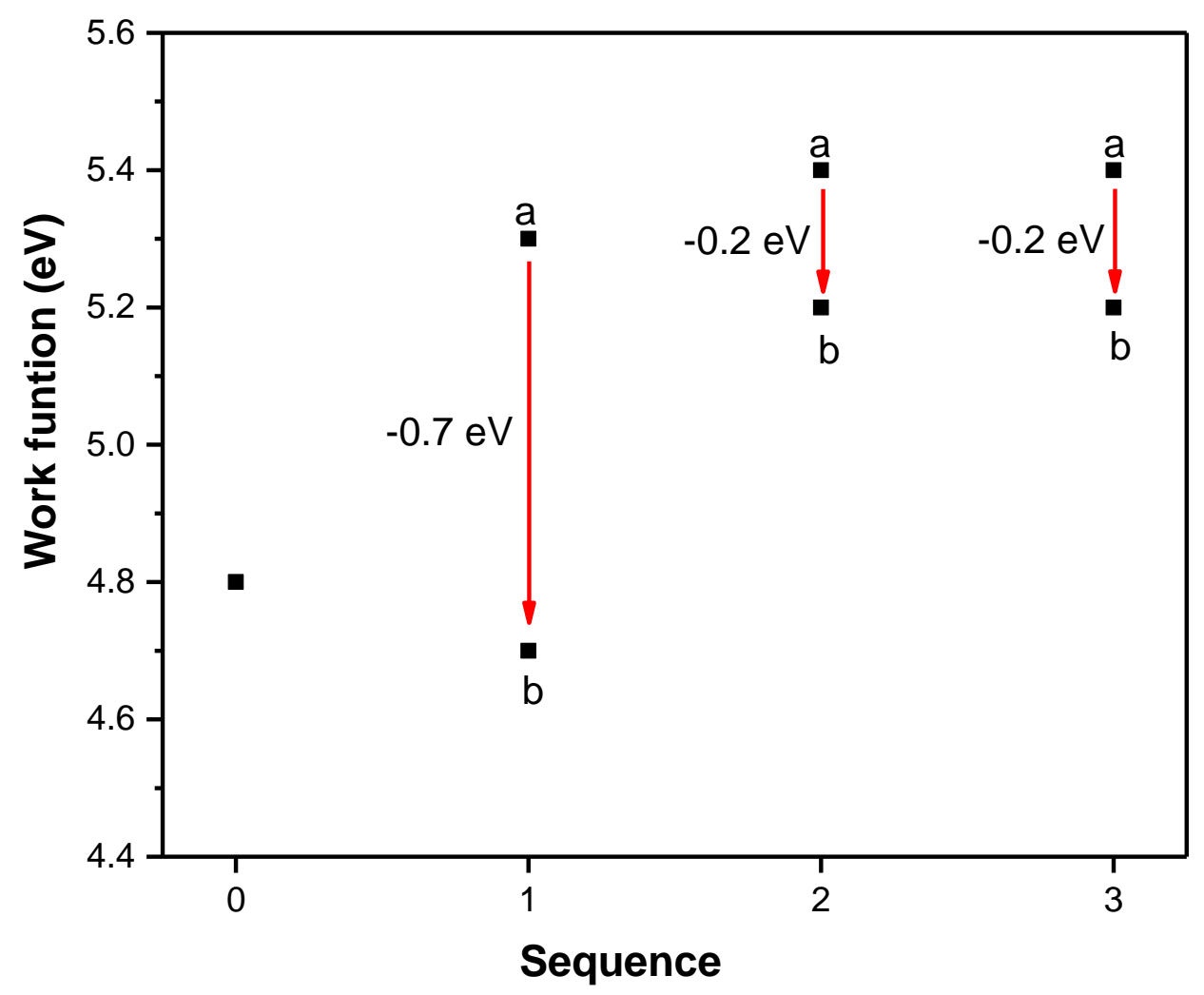


Figure 10: work function degradation in vacuum for different plasma processing experiments mentioned in table 1 . Subsequent plasma processing was found beneficial for maintaining higher work function over a longer period of time.

Because, migration process depends exponentially on temperature [28], no hydrocarbons recovery at top surface is expected after a cavity is cooled-down to cryogenic temperature. Hence, immediate cool-down of a cavity after the plasma processing is desirable. However, the cool down of a cryomodule takes hours. Based on the results from surface studies, it was found that a few cycles of plasma processing with waiting periods in between is beneficial to mitigate the recovery of hydrocarbons at top surface and to sustain higher work function in vacuum at room temperature. Therefore this methodology could allow suitable waiting time after the plasma processing until the cryomodule is cold.

\section{CONCLUSION}

Detailed studies of plasma processed niobium surface were conducted. The results showed that plasma processing using reactive oxygen is very effective to remove hydrocarbons from the niobium surface. Studies showed that such plasma processing increases the work function of the niobium surface systematically in the range of 0.5 to $1 \mathrm{eV}$. SIMS studies revealed that after the top surface is plasma cleaned, near-surface hydrocarbons can migrate to the top surface when a sample is kept in vacuum at room temperature. Removal and recovery of hydrocarbons were also observed in plasma processed cavities. Scanning kelvin probe measurements showed that the work function of the niobium surface correlates with the level of hydrocarbons at top surface. A few plasma processing cycles with waiting periods in between was found to be an effective technique to remove top surface and near surface hydrocarbons and to increase and sustain the work function. This technique was successfully applied to cavities at room temperature and improvement in their if performance was demonstrated by cold tests [12].

\section{ACKNOWLEDGEMENT}

The authors express their gratitude towards Department of Energy (DOE), USA for funding support of the work presented here under the contract DE-AC05-00OR22725. 


\section{References}

[1] Jay Benesch "Field Emission in CEBAF's Superconducting RF Cavities and Implications for Future Accelerators" arXiv:physics/0606141v2

[2] S. Aderhold et. al. "Analysis Of Degraded Cavities In Prototype Modules For The European XFEL" Proceedings of SRF2015, Whistler, BC, Canada

[3] S-H Kim "Status of the SNS Superconducting Linac and Future Plan" Journal of the Korean Physical Society, Vol. 52, No. 3, March 2008, pp. 714-720 (2008)

[4] Hasan Padamsee, "RF Superconductivity: Volume II" John Wiley \& Sons.

[5] J. Knobloch, "Field emission and thermal breakdown in superconducting niobium cavities for accelerators," IEEE Trans. Appl. Supercond. 9(2), 1016-1022 (1999).

[6] J. Halbritter, "Enhanced electron emission and its reduction by electron and ion impact," IEEE Trans. Electr. Insul. El-18(3), 252-261 (1983).

[7] M. Doleans et. al. "Plasma processing $R \& D$ for the SNS superconducting linac if cavities", SRF2013, Paris, France (2013).

[8] S. M. Irving, "A plasma oxidation process for removing photoresist films," Solid State Technol. 1971, 47.

[9] J. W. Coburn, "Surface processing with partially ionized plasma," IEEE Trans. Plasma Sci. 19(6), 1048 (1991).

[10] J. Upadhyay et. al. "Plasma processing of large curved surfaces for superconducting if cavity modification" Physical Review Special Topics Accelerators And Beams 17, 122001 (2014).

[11] M. Rašković et. al. "Plasma treatment of bulk niobium surface for superconducting rf cavities: Optimization of the experimental conditions on flat samples" Physical Review Special Topics - Accelerators And Beams 13, 112001 (2010).

[12] S-H Kim et al., "R\&D Status for In-Situ Plasma Surface Cleaning of SRF Cavities at Spallation Neutron Source", Proceedings of 2011 Particle Accelerator Conference, New York, NY, USA.

[13] M. Doleans et. al. "In situ plasma processing to increase the accelerating gradients of superconducting radio-frequency cavities", Nuclear Instruments and Methods in Physics Research Section A, 812, 50-59, (2016).

[14] P. V. Tyagi et. al. "Surface Studies of Plasma Processed Nb Samples" Proceedings of the Workshop on RF Superconductivity, Vancouver, Canada (2015).

[15] J. W. Wang and G. A. Loew "Field Emission and RF Breakdown in High-Gradient Room-Temperature Linac Structures" SLAC-PUB-7684 (1997).

[16] Scanning Kelvin Probe - SKP5050, Kelvin Probe technology webinar, http://www.kelvinprobe.info/

[17] I. D. Baikie et al, "Low cost PC based scanning Kelvin probe" Rev. Sci. Instrum., Vol. 69, No. 11 Nov '98. 
[18] H. B. Michaelson "The work function of the elements and its periodicity" J. Appl. Phys. 48, 4729 (1977)

[19] Dacca, A et. al., "XPS characterization of niobium for RF cavities" Proceedings of the Workshop on RF Superconductivity, Abano Terme (Padova), Italy (1997).

[20] Jian Wang and Shao-Qing Wang "Surface energy and work function of fcc and bcc crystals: Density functional study" Surface Science, 630, 216-224 (2014).

[21] W. Gao et. al., "NbO as gate electrode for n-channel metal-oxide-semiconductor field-effect-transistors" Applied Physics Letters 84, 4666 (2004).

[22] John C. Vikerman and lan S. Gilmore "Surface analysis: the principle techniques" John Wiley \& Sons.

[23] Scientific Analysis Instruments Limited, MiniSIMS TOF/alpha, user manual 1.2.

[24] Donald C. Zipperian, "Metallographic handbook" PACE Technologies Tucson, Arizona USA.

[25] SPI Plasma Prep Jr, http://www.2spi.com/

[26] W. Umrath et al. "Fundamentals of vacuum technology" Oerlikon Leybold vacuum 00.200.02, Kat.-Nr. 19990 (2007)

[27] P. V. Tyagi et. al. "Plasma processing of Nb surfaces for SRF cavities" Proceedings of 27th International Linear Accelerator Conference (LINAC14, Aug 31Sept 6, 2014, Geneva, Switzerland.

[28] James F. Shackelford "Introduction to Materials Science for Engineers" ISBN-10: 0133826651 